\title{
ON ALMOST COSYMPLECTIC MANIFOLDS
}

\author{
By ZBigniew OlsZaK
}

\section{$\S 1$. Introduction.}

The purpose of the present paper is to study the structure of almost cosymplectic manifolds. $\S 2$ presents the basic definitions and some preliminary properties of an almost cosymplectic structure. Examples of such structures are given in $\S 3$. In $\S 4$ we state many important curvature identities for almost cosymplectic manifolds. In $\S 5$ we give certain sufficient conditions for an almost contact metricstructure to be almost cosymplectic. Basing on the identities from $\S 4$ we prove in $\S 6$ that almost cosymplectic manifolds of non-zero constant sectional curvature do not exist in dimensions greater than three. However it is known (cf. [2]) that such manifolds of zero sectional curvature (i. e. locally flat) exist and they are cosymplectic. Moreover we give certain restrictions on the scalar curvature of almost cosymplectic manifolds which are conformally flat or of constant $\phi$-sectional curvature.

All manifolds considered in this paper are assumed to be connected and of class $C^{\infty}$. All tensor fields, including differential forms, are of class $C^{\infty}$. The notation and terminology will be the same as that employed in [2].

\section{§. Preliminaries.}

Let $(M, \phi, \xi, \eta, g)$ be a $(2 n+1)$-dimensional almost contact metric manifold, that is, $M$ is a differentiable manifold and $(\phi, \xi, \eta, g)$ an almost contact metric structure on $M$, formed by tensor fields $\phi, \xi$, $\eta$ of type $(1,1),(1,0),(0,1)$, respectively, and a Riemannian metric $g$ such that

$$
\begin{array}{ll}
\phi^{2}=-I+\eta \otimes \xi, & \phi \xi=0, \quad \eta \circ \phi=0, \quad \eta(\xi)=1, \\
\eta(X)=g(X, \xi), & g(\phi X, \phi Y)=g(X, Y)-\eta(X) \eta(Y) .
\end{array}
$$

On such manifold we may always define a 2 -form $\Phi$ by $\Phi(X, Y)=g(\phi X, Y)$. $(M, \phi, \xi, \eta, g)$ is said to be an almost cosymplectic manifold (cf. [2]) if the forms $\Phi$ and $\eta$ are closed, i. e. $d \Phi=0$ and $d \eta=0$, where $d$ is the operator of exterior differentiation. In particular, if the almost contact structure of an almost cosymplectic manifold is normal, then it is said to be a cosymplectic manifold (cf. [1]).

Received July 9, 1979 
As it is known, an almost contact metric structure is cosymplectic if and only if both $\nabla \eta$ and $\nabla \Phi$ vanish, where $\nabla$ is the covariant differentiation with respect to $g$.

LEMMa 2.1. Let $(M, \phi, \xi, \eta, g)$ be an almost contact metric manfold. If 2form $\Phi$ is closed, then

$$
\begin{aligned}
& \left(\nabla_{\phi X} \Phi\right)(\phi Y, Z)+\left(\nabla_{X} \Phi\right)(Y, Z)-\eta(X)\{d \eta(\phi Y, Z)+d \eta(Y, \phi Z)\} \\
& +\eta(Y)\left\{d \eta(\phi Z, X)-1 / 2\left(\mathcal{L}_{\xi} g\right)(Z, \phi X)\right\}+\eta(Z)\{d \eta(X, \phi Y)-d \eta(\phi X, Y)\}=0,
\end{aligned}
$$

where $\mathcal{L}$ is Lie differentiation.

Proof. Because $\Phi$ is closed, we have $\left(\nabla_{X} \Phi\right)(Y, Z)+\left(\nabla_{Y} \Phi\right)(Z, X)+\left(\nabla_{Z} \Phi\right)(X, Y)$ $=0$. This implies

$$
\begin{aligned}
& \left(\nabla_{X} \Phi\right)(Y, Z)+\left(\nabla_{Y} \Phi\right)(Z, X)+\left(\nabla_{Z} \Phi\right)(X, Y) \\
+ & \left(\nabla_{Z} \Phi\right)(\phi X, \phi Y)+\left(\nabla_{\phi X} \Phi\right)(\phi Y, Z)+\left(\nabla_{\phi Y} \Phi\right)(Z, \phi X) \\
- & \left(\nabla_{X} \Phi\right)(\phi Y, \phi Z)-\left(\nabla_{\phi Y} \Phi\right)(\phi Z, X)-\left(\nabla_{\phi Z} \Phi\right)(X, \phi Y) \\
+ & \left(\nabla_{y} \Phi\right)(\phi Z, \phi X)+\left(\nabla_{\phi Z} \Phi\right)(\phi X, Y)+\left(\nabla_{\phi X} \Phi\right)(Y, \phi Z)=0 .
\end{aligned}
$$

The following relations are valid in any almost contact metric manifold and are easy to obtain

$$
\begin{aligned}
& \left(\nabla_{X} \Phi\right)(\phi Y, \phi Z)+\left(\nabla_{X} \Phi\right)(Y, Z)=\eta(Y)\left(\nabla_{X} \eta\right)(\phi Z)-\eta(Z)\left(\nabla_{X} \eta\right)(\phi Y), \\
& \left(\nabla_{X} \Phi\right)(\phi Y, Z)-\left(\nabla_{X} \Phi\right)(Y, \phi Z)=\eta(Y)\left(\nabla_{X} \eta\right)(Z)+\eta(Z)\left(\nabla_{X} \eta\right)(Y) .
\end{aligned}
$$

In view of (2.3) and (2.4) the equality (2.2) reduces to (2.1), if we recall that $d \eta(X, Y)=1 / 2\left\{\left(\nabla_{X} \eta\right)(Y)-\left(\nabla_{Y} \eta\right)(X)\right\} \quad$ and $\left(\mathcal{L}_{\xi} g\right)(X, Y)=g\left(\nabla_{X} \xi, Y\right)+g\left(\nabla_{Y} \xi, X\right)=$ $\left(\nabla_{X} \eta\right)(Y)+\left(\nabla_{Y} \eta\right)(X)$. The proof is complete.

Suppose that $(M, \phi, \xi, \eta, g)$ is almost cosymplectic. Thus, the relations

$$
\begin{aligned}
& \left(\nabla_{X} \eta\right)(Y)-\left(\nabla_{Y} \eta\right)(X)=0, \\
& \left(\nabla_{X} \Phi\right)(Y, Z)+\left(\nabla_{Y} \Phi\right)(Z, X)+\left(\nabla_{Z} \Phi\right)(X, Y)=0
\end{aligned}
$$

hold good.

LEMMA 2.2. In an almost cosymplectic manifold we have

$$
\left(\nabla_{\phi X} \phi\right) \phi Y+\left(\nabla_{X} \phi\right) Y-\eta(Y) \nabla_{\phi X} \xi=0 .
$$

Proof. In our case $d \eta=0$ and $\left(\mathcal{L}_{\xi} g\right)(Z, \phi X)=2\left(\nabla_{\phi X} \eta\right)(Z)$, by (2.5). Therefore (2.1) gives

$$
\left(\nabla_{\phi X} \Phi\right)(\phi Y, Z)+\left(\nabla_{X} \Phi\right)(Y, Z)-\eta(Y)\left(\nabla_{\phi X} \eta\right)(Z)=0
$$


which leads to (2.7), completing the proof.

Now we give certain consequences of (2.7), which together with (2.7) play very important role in $\S 4$. First, by

$$
\left(\nabla_{\phi X} \phi\right) \phi Y=\left(\nabla_{\phi X} \eta\right)(Y) \xi+\eta(Y) \nabla_{\phi X} \xi-\phi\left(\nabla_{\phi X} \phi\right) Y
$$

we obtain from $(2.7)$

$$
\phi\left(\nabla_{\phi X} \phi\right) Y-\left(\nabla_{X} \phi\right) Y-\left(\nabla_{\phi X} \eta\right)(Y) \xi=0 .
$$

Operating $\phi$ onto (2.8) we get

$$
\left(\nabla_{\phi X} \phi\right) Y+\phi\left(\nabla_{X} \phi\right) Y+\left(\nabla_{\phi X} \eta\right)(\phi Y) \xi=0 .
$$

(2.7) for $X=\xi, Y=\xi$ results

$$
\begin{gathered}
\nabla_{\xi} \phi=0, \\
\nabla_{\phi X} \xi=-\phi \nabla_{X} \xi,
\end{gathered}
$$

respectively. Substituting $X=\xi$ in $(2.11)$, we see that $\phi \nabla_{\xi} \xi=0$, which yields

$$
\nabla_{\xi} \xi=0 \text {. }
$$

On the other hand, (2.11) gives

$$
\left(\nabla_{\phi X} \eta\right)(Y)=\left(\nabla_{X} \eta\right)(\phi Y)
$$

hence it follows that

$$
\left(\nabla_{\phi X} \eta\right)(\phi Y)=-\left(\nabla_{X} \eta\right)(Y)
$$

\section{$\S 3$. Examples of almost cosymplectic manifolds.}

The simplest examples of almost cosymplectic manifolds may be constructed as follows. Let $(N, J, G)$ be an almost Kählerian manifold, that is, $N$ is a $2 n$ dimensional manifold, $J$ is an almost complex structure and $G$ is a Riemannian metric on $N$ such that $J^{2}=-I, G(J X, J Y)=G(X, Y)$ and $d F=0$, where $F$ is 2form defined on $N$ by $F(X, Y)=G(J X, Y)$. Let $R$ be real line, and let $G_{0}$ be a Riemannian metric, $\xi_{0}$ a vector field and $\eta_{0}$ a 1 -form on $\boldsymbol{R}$ such that $\eta_{0}\left(\xi_{0}\right)=1$ and $G_{0}\left(\xi_{0}, \xi_{0}\right)=1$. Consider the manifold $M=N \times \boldsymbol{R}$. Let $\left(X_{1}, X_{2}\right)$ denote a tangent vector to $M$, where $X_{1}$ is a tangent vector to $N$ and $X_{2}$ is a tangent vector to $\boldsymbol{R}$. Define a tensor field $\phi$ of type $(1,1)$, a vector field $\xi$ and a 1 -form $\eta$ on $M$ taking $\phi\left(X_{1}, X_{2}\right)=\left(J X_{1}, 0\right), \xi=\left(0, \xi_{0}\right)$ and $\eta\left(\left(X_{1}, X_{2}\right)\right)=\eta_{0}\left(X_{2}\right)$. Define also a Riemannian metric $g$ on $M$ as the product of the metrics $G$ and $G_{0}$. Then $(\phi, \xi, \eta, g)$ is clearly an almost cosymplectic structure on $M$. In like manner one can construct an almost cosymplectic structure on $N \times S^{1}$, where $S^{1}$ is a circle. In both these cases the structure vector field is parallel with respect to the Rie- 
mannian connection.

In the sequel we state almost cosymplectic structures with non-parallel vector field $\xi$ on certain Lie groups in every odd dimension.

Let $L$ be a $(2 n+1)$-dimensional real vector space and choose a basis $\left\{e_{0}, e_{1}\right.$, $\left.\cdots, e_{2 n}\right\}$ of $L$. Let $A_{1}, \cdots, A_{2 n}$ be constants such that $A_{1}^{2}+\cdots+A_{2 n}^{2}>0$. Define a two-linear mapping $[\cdot, \cdot]: L \times L \rightarrow L$, taking for the basis vectors

$$
\begin{aligned}
& {\left[e_{0}, e_{\imath}\right]=-\left[e_{\imath}, e_{0}\right]=-A_{i} e_{i}-A_{\imath+n} e_{\imath+n},} \\
& {\left[e_{0}, e_{\imath+n}\right]=-\left[e_{\imath+n}, e_{0}\right]=-A_{\imath+n} e_{i}+A_{i} e_{\imath+n}}
\end{aligned}
$$

for $\imath=1, \cdots, n$ and $\left[e_{\jmath}, e_{k}\right]=0$ in other cases. Observe that $[\cdot, \cdot]$ is antisymmetric and satisfies the Jacobi identity. Thus, $L$ is a $(2 n+1)$-dimensional Lie algebra with respect to $[\cdot, \cdot]$.

Consider a connected Lie subgroup $M$ of general linear group $G L(k, \boldsymbol{R})$, for certain $k$, such that the Lie algebra $L M$ of $M$ is isomorphic with $L$. Let $\sigma$ : $L \rightarrow L M$ be the isomorphism. Let $\left\{E_{0}, E_{1}, \cdots, E_{2 n}\right\}$ be the basis of $L M$ formed by left invariant vector fields on $M$ such that $E_{\jmath}=\sigma\left(e_{j}\right)$ for $\jmath=0,1, \cdots, 2 n$. Then

$$
\begin{aligned}
& {\left[E_{0}, E_{\imath}\right]=-A_{\imath} E_{i}-A_{\imath+n} E_{\imath+n},} \\
& {\left[E_{0}, E_{\imath+n}\right]=-A_{\imath+n} E_{i}+A_{\imath} E_{\imath+n}}
\end{aligned}
$$

for $i=1, \cdots, n$ and $\left[E_{\jmath}, E_{k}\right]=0$ in other cases. Define a left invariant Riemannian metric $g$ on $M$ by $g\left(E_{\jmath}, E_{k}\right)=\delta_{j k}, \jmath, k=0,1, \cdots, 2 n$. Define a left invariant linear connection $\nabla$ on $M$ by

$$
\begin{aligned}
& \nabla_{E_{i}} E_{0}=A_{\imath} E_{i}+A_{\imath+n} E_{\imath+n}, \nabla_{E_{i}} E_{\imath}=-A_{\imath} E_{0}, \nabla_{E_{\imath}} E_{\imath+n}=-A_{\imath+n} E_{0}, \\
& \nabla_{E_{\imath+n}} E_{0}=A_{\imath+n} E_{i}-A_{\imath} E_{\imath+n}, \nabla_{E_{\imath+n}} E_{\imath}=-A_{\imath+n} E_{0}, \nabla_{E_{\imath+n}} E_{\imath+n}=A_{\imath} E_{0},
\end{aligned}
$$

for $\imath=1, \cdots, n$ and $\nabla_{E_{j}} E_{k}=0$ in other cases. It is easy to verify that $\nabla$ is metric with respect to $g$ and symmetric, that is, $\nabla$ is the Riemannian connection with respect to $g$.

Define a 1 -form $\eta$ and a $(1,1)$-tensor field $\phi$ on $M$ by $\eta\left(E_{j}\right)=\delta_{0 \jmath}$, for $\jmath=0,1$, $\cdots, 2 n$, and $\phi E_{0}=0, \phi E_{\imath}=E_{\imath+n}, \phi E_{\imath+n}=-E_{\imath}$, for $\imath=1, \cdots, n$. Suppose also $\xi=E_{0}$. Then $(\phi, \xi, \eta, g)$ is an almost contact metric structure on $M$. We show that $(\phi, \xi, \eta, g)$ is an almost cosymplectic structure. First one can find that

$$
2 d \eta\left(E_{\jmath}, E_{k}\right)=g\left(\nabla_{E_{j}} E_{0}, E_{k}\right)-g\left(\nabla_{E_{k}} E_{0}, E_{j}\right)=0,
$$

for $j, k=0,1, \cdots, 2 n$, i. e. (2.5) holds good. Further, from our definitions follow the equalities

$$
\begin{aligned}
& \left(\nabla_{E_{i}} \phi\right) E_{0}=A_{\imath+n} E_{i}-A_{\imath} E_{\imath+n},\left(\nabla_{E_{i}} \phi\right) E_{\imath}=-A_{\imath+n} E_{0},\left(\nabla_{E_{\imath}} \phi\right) E_{\imath+n}=A_{\imath} E_{0}, \\
& \left(\nabla_{E_{\imath+n}} \phi\right) E_{0}=-A_{\imath} E_{i}-A_{\imath+n} E_{\imath+n},\left(\nabla_{E_{\imath+n}} \phi\right) E_{\imath}=A_{\imath} E_{0},\left(\nabla_{E_{\imath+n}} \phi\right) E_{\imath+n}=A_{\imath+n} E_{0},
\end{aligned}
$$


for $\imath=1, \cdots, n$ and $\left(\nabla_{E_{j}} \phi\right) E_{k}=0$ in other cases. In virtue of these relations, we verify that

$$
3(d \Phi)\left(E_{\jmath}, E_{k}, E_{l}\right)=g\left(\left(\nabla_{E_{j}} \phi\right) E_{k}, E_{l}\right)+g\left(\left(\nabla_{E_{k}} \phi\right) E_{l}, E_{\jmath}\right)+g\left(\left(\nabla_{E_{l}} \phi\right) E_{\jmath}, E_{k}\right)=0,
$$

for $\}, k, l=0,1, \cdots, 2 n$, i. e. (2.6) holds good. Finally, because of $A_{1}^{2}+\cdots+A_{2 n}^{2}>0$, $\nabla \xi \neq 0$ and $\nabla \phi \neq 0$.

\section{$\S 4$. Curvature identities.}

Assume that $(M, \phi, \xi, \eta, g)$ is an almost cosymplectic manifold. We denote by $R$ the curvature tensor, i. e. $R_{X Y Z W}=g\left(R_{X Y} Z, W\right)$, where $R_{X Y}=\left[\nabla_{X}, \nabla_{Y}\right]-\nabla_{[X, Y]}$ is the curvature transformation. Let $S$ be the Ricci tensor, and $S^{*}$ the Ricci * tensor defined by $S^{*}(X, Y)=\sum_{\imath=0}^{2 n} R_{E_{\imath} X \phi Y \phi E_{i}}$, where $\left\{E_{0}, E_{1}, \cdots, E_{2 n}\right\}$ is an orthonormal frame. Let $r$ be the scalar curvature and $r^{*}$ the scalar $*$ curvature defined by $r^{*}=\sum_{\imath=0}^{2 n} S^{*}\left(E_{\imath}, E_{\imath}\right)$.

THEOREM 4.1. Any almost cosymplectic manifold satısfies the following identities

$$
\begin{gathered}
R_{X Y Z W}-R_{X Y \phi Z \phi W}-R_{\phi X \phi Y Z W}+R_{\phi X \phi Y \phi Z \phi W}+R_{\phi X Y \phi Z W}+R_{\phi X Y Z \phi W} \\
+R_{X \phi Y \phi Z W}+R_{X \phi Y Z \phi W}-2 \sum_{\imath=0}^{2 n}\left(\nabla_{E_{\imath}} \Phi\right)(X, Y)\left(\nabla_{E_{\imath}} \Phi\right)(Z, W)=0 \\
S(Y, Z)+S(\phi Y, \phi Z)-S^{*}(Y, Z)-S^{*}(Z, Y) \\
-\frac{1}{2}\left(R_{\xi \phi Y \phi Z \xi}+R_{\xi Y Z \xi}\right)+\sum_{\imath=0}^{2 n} g\left(\left(\nabla_{E_{\imath}} \phi\right) Y,\left(\nabla_{E_{\imath}} \phi\right) Z\right)=0, \\
r-r^{*}-S(\xi, \xi)+\frac{1}{2}|\nabla \phi|^{2}=0 .
\end{gathered}
$$

Proof. Relation (2.9) in virtue of (2.14) may be rewritten in the following form

$$
\nabla_{\phi Y} \phi Z-\phi \nabla_{\phi Y} Z+\phi \nabla_{Y} \phi Z+\nabla_{Y} Z-Y(\eta(Z)) \xi=0
$$

Hence by the covariant differentiation we derive

$$
\nabla_{X} \nabla_{\phi Y} \phi Z-\nabla_{X} \phi \nabla_{\phi Y} Z+\nabla_{X} \phi \nabla_{Y} \phi Z+\nabla_{X} \nabla_{Y} Z-X(Y(\eta(Z))) \xi-Y(\eta(Z)) \nabla_{X} \xi=0 .
$$

Taking $\phi X$ instead of $X$ in (4.5) and using (4.4) and (2.11) we obtain 


$$
\begin{aligned}
& \nabla_{\phi X} \nabla_{\phi Y} \phi Z-\phi \nabla_{\phi X} \nabla_{\phi Y} Z-\nabla_{X} \nabla_{Y} \phi Z+\nabla_{X} \nabla_{\phi Y} Z+\phi \nabla_{\phi X} \nabla_{Y} \phi Z+\nabla_{\phi X} \nabla_{Y} Z \\
& \quad+\phi D_{X} \phi \nabla_{\phi Y} Z-\phi \nabla_{X} \phi \nabla_{Y} \phi Z-X\left(\eta\left(\nabla_{\phi Y} Z\right)\right) \xi+X\left(\eta\left(\nabla_{Y} \phi Z\right)\right) \xi \\
& \quad-(\phi X)(Y(\eta(Z))) \xi+Y(\eta(Z)) \phi \nabla_{X} \xi=0 .
\end{aligned}
$$

On the other hand operating $\phi$ onto (4.5) we have

$$
\phi \nabla_{X} \nabla_{\phi Y} \phi Z-\phi \nabla_{X} \phi \nabla_{\phi Y} Z+\phi \nabla_{X} \phi \nabla_{Y} \phi Z+\phi V_{X} \nabla_{Y} Z-Y(\eta(Z)) \phi \nabla_{X} \xi=0 .
$$

Adding the two last equalities we get

$$
\begin{aligned}
& \nabla_{\phi X} \nabla_{\phi Y} \phi Z-\phi \nabla_{\phi X} \nabla_{\phi Y} Z-\nabla_{X} \nabla_{Y} \phi Z+\phi \nabla_{X} \nabla_{Y} Z+\phi \nabla_{\phi X} \nabla_{Y} \phi Z+\nabla_{\phi X} \nabla_{Y} Z \\
& +\phi \nabla_{X} \nabla_{\phi Y} \phi Z+\nabla_{X} \nabla_{\phi Y} Z-X\left(\eta\left(\nabla_{\phi Y} Z\right)\right) \xi-(\phi X)(Y(\eta(Z))) \xi+X\left(\eta\left(\nabla_{Y} \phi Z\right)\right) \xi=0 .
\end{aligned}
$$

In the sequel we fix a point $m \in M$ and assume that $X, Y, Z, W$ are covariant constant at $m$. Then the antisymmetrization of (4.6) with respect to $X$ and $Y$ and the projection of the resulting equation onto $\phi W$ lead to

$$
\begin{aligned}
& R_{\phi X \phi Y \phi Z \zeta W}-R_{\phi X \phi Y Z W}-R_{X Y \phi Z \phi W}+R_{X Y Z W} \\
+ & R_{\phi X Y \phi Z W}+R_{\phi X Y Z \phi W}+R_{X \phi Y \phi Z W}+R_{X \phi Y Z \phi W} \\
+ & g\left(V_{[\phi X, \phi Y]} \phi Z, \phi W\right)+g\left(\nabla_{[\phi X, Y]+[X, \phi Y]} \phi Z, W\right) \\
+ & \eta(W)\left\{R_{\phi X \phi Y Z \xi}-R_{X Y Z \xi}-R_{\phi X Y \phi Z \xi}-R_{X \phi Y \phi Z \xi}-\eta\left(V_{[\phi X, Y]+[X, \phi Y]} \phi Z\right)\right\}=0 .
\end{aligned}
$$

From (2.6) it follows that $\left(\nabla_{X} \phi\right) Y-\left(\nabla_{Y} \phi\right) X=-\sum_{\imath=0}^{2 n}\left(\nabla_{E_{\imath}} \Phi\right)(X, Y) E_{\imath}$. Therefore and by (2.9) and (2.5) we find

$$
\begin{aligned}
& {[\phi X, \phi Y]=\left(\nabla_{\phi X} \phi\right) Y-\left(\nabla_{\phi Y} \phi\right) X=-\phi\left\{\left(\nabla_{X} \phi\right) Y-\left(\nabla_{Y} \phi\right) X\right\}=\sum_{\imath=0}^{2 n}\left(\nabla_{E_{\imath}} \Phi\right)(X, Y) \phi E_{\imath},} \\
& {[\phi X, Y]+[X, \phi Y]=\left(\nabla_{X} \phi\right) Y-\left(\nabla_{Y} \phi\right) X=-\sum_{\imath=0}^{2 n}\left(\nabla_{E_{\imath}} \Phi\right)(X, Y) E_{\imath} .}
\end{aligned}
$$

These identities and (4.4) imply

$$
\begin{aligned}
& g\left(\nabla_{[\phi X, \phi Y]} \phi Z, \phi W\right)=\sum_{\imath=0}^{2 n}\left(\nabla_{E_{\imath}} \Phi\right)(X, Y) g\left(\nabla_{\phi E_{i}} \phi Z, \phi W\right) \\
& =-\sum_{\imath=0}^{2 n}\left(\nabla_{E_{\imath}} \Phi\right)(X, Y) g\left(\phi \nabla_{E_{i}} \phi Z, \phi W\right) \\
& =-\sum_{\imath=0}^{2 n}\left(\nabla_{E_{\imath}} \Phi\right)(X, Y)\left\{\left(\nabla_{E_{\imath}} \Phi\right)(Z, W)-\eta\left(\nabla_{E_{i}} \phi Z\right) n(W)\right\}, \\
& g\left(\nabla_{[\phi X X, Y]+[X, \phi Y]} \phi Z, W\right)=-\sum_{\imath=0}^{2 n}\left(\nabla_{E_{\imath}} \Phi\right)(X, Y)\left(\nabla_{E_{\imath}} \Phi\right)(Z, W),
\end{aligned}
$$




$$
\eta\left(\nabla_{[\phi X, Y]+[X, \phi Y]} \phi Z\right)=-\sum_{\imath=0}^{2 n}\left(\nabla_{E_{\imath}} \Phi\right)(X, Y) \eta\left(\nabla_{E_{\imath}} \phi Z\right) .
$$

Moreover, using (2.6), (2.10) and (2.11), we find

$$
\eta\left(\nabla_{E_{i}} \phi Z\right)=g\left(\left(\nabla_{E_{i}} \phi\right) Z, \xi\right)=-g\left(\left(\nabla_{Z} \phi\right) \xi, E_{\imath}\right)=g\left(\phi \nabla_{Z} \xi, E_{\imath}\right)=-g\left(\nabla_{\dot{\phi} Z} \hat{\xi}, E_{\imath}\right) .
$$

If we use the above identities in (4.7), then we obtain

$$
\begin{aligned}
& R_{\phi X \phi Y \phi Z \phi W}-R_{\phi X \phi Y Z W}-R_{X Y \phi Z \phi W}+R_{X Y Z W} \\
& +R_{\phi X Y \phi Z W}+R_{\phi X Y Z \phi W}+R_{X \phi Y \phi Z W}+R_{X \phi Y Z \phi W} \\
& +\eta(W)\left\{R_{\phi X \phi Y Z \xi}-R_{X Y Z \xi}-R_{\phi X Y \phi Z \xi}-R_{X \phi Y \phi Z \xi}-2\left(\nabla_{\nabla_{\phi Z} \xi} \Phi\right)(X, Y)\right\} \\
& -2 \sum_{\imath=0}^{2 n}\left(\nabla_{E_{\imath}} \Phi\right)(X, Y)\left(\nabla_{E_{\imath}} \Phi\right)(Z, W)=0 .
\end{aligned}
$$

Symmetrizing of (4.8) with respect to $Z$ and $W$, and next taking $W=\xi$ in the resulting equality we find

$$
R_{\phi X \phi Y Z \xi}-R_{X Y Z \xi}-R_{\phi X Y \phi Z \xi}-R_{X \phi Y \dot{\phi} \xi \xi}-2\left(\nabla_{\nabla_{\phi Z} \xi} \Phi\right)(X, Y)=0 .
$$

In view of the above identity, (4.8) implies (4.1).

Now we choose a $\phi$-basis, i.e. an orthonormal frame $\left\{E_{0}, E_{1}, \cdots, E_{2 n}\right\}$ such that $E_{0}=\xi$ and $E_{\imath+n}=\phi E_{\imath}$, for $\imath=1, \cdots, n$. Taking $X=W=E_{\jmath}$ into (4.1) and summing over $\jmath=0,1, \cdots, 2 n$, we obtain (4.2). Substituting $Y=Z=E_{k}$ into (4.2) and summing over $k=0,1, \cdots, 2 n$ we get (4.3). This completes the proof.

THEOREM 4.2. For any almost cosymplectic manifold we have

$$
\begin{gathered}
R_{X Y \phi Z \xi}-R_{\phi X \phi Y \phi Z \xi}-R_{\phi X Y Z \xi}-R_{X \phi Y Z \xi}-2\left(\nabla_{\nabla Z \xi} \Phi\right)(X, Y)=0, \\
R_{\xi Y Z \xi}+R_{\xi \phi Y \phi Z \xi}+2 g\left(\nabla_{Y} \xi, \nabla_{Z} \xi\right)=0, \\
S(\xi, \xi)+|\nabla \xi|^{2}=0 .
\end{gathered}
$$

Proof. We have already known that

$$
R_{X Y Z \xi}-R_{\phi X \phi Y Z \xi}+R_{\phi X Y \phi Z \xi}+R_{X \phi Y \phi Z \xi}+2\left(\nabla_{\nabla_{\phi Z} \xi} \Phi\right)(X, Y)=0 .
$$

If we take $\phi Z$ instead of $Z$ in (4.12) and recall (2.12), then we find (4.9). For $X=\xi$, the relation (4.12) yields

$$
R_{\xi Y Z \xi}+R_{\xi \phi Y \dot{\phi} \xi}+2\left(\nabla_{\nabla_{\phi \zeta \xi}} \Phi\right)(\xi, Y)=0 .
$$

But by (2.11) and (2.5) we have

$$
\left(\nabla_{\nabla_{\phi Z} \xi} \Phi\right)(\xi, Y)=-g\left(\phi \nabla_{\nabla_{\phi Z} \xi} \xi, Y\right)=g\left(\nabla_{\nabla_{Z} \xi} \xi, Y\right)=g\left(\nabla_{Y} \xi, \nabla_{Z} \xi\right),
$$

which together with (4.13) implies (4.10). Now for a choosen $\phi$-basis we take 
$Y=Z=E$, into (4.10) and sum over $\jmath=0,1, \cdots, 2 n$. Then we obtain (4.11), which completes the proof.

\section{$\S 5$. Certain sufficient conditions.}

First of all we state the necessary denotations.

Let $(M, \dot{\phi}, \xi, \eta, g)$ be an almost contact metric manifold. Let $\left\{E_{0}, E_{1}, \cdots, E_{2 n}\right\}$ be an orthonormal frame of $T_{p}(M), p \in M$. By the same letters we denote local extension vector fields of this frame, which are orthonormal and covariant constant at $p$. If $V$ is a vector field on $M$, then its divergence is given by

$$
\operatorname{div} V=\operatorname{trace}\left\{X \rightarrow \nabla_{X} V\right\}=\sum_{\imath=0}^{2 n} g\left(\nabla_{E_{\imath}} V, E_{\imath}\right) .
$$

If $\omega$ is a differential $r$-form on $M$, then the codifferential of $\omega$ is defined by

$$
\delta \omega\left(Y_{1}, \cdots, Y_{r-1}\right)=-\sum_{\imath=0}^{2 n}\left(\nabla_{E_{\imath}} \omega\right)\left(E_{\imath}, Y_{1}, \cdots, Y_{r-1}\right) .
$$

A differential form is said to be harmonic, if it is zero of the operators $d$ and $\delta$.

LEMMA 5.1.

$$
\operatorname{div}\left(\nabla_{\xi} \xi+\delta \eta \xi\right)=S(\xi, \xi)+|\nabla \xi|^{2}-(\delta \eta)^{2}-2|d \eta|^{2} .
$$

Proof is given in local coordinates in [4], p. 41.

LEMMA 5.2. If $V=\sum_{\imath=0}^{2 n} \phi\left(\nabla_{E_{i}} \phi\right) E_{\imath}$ and $U=\sum_{\imath=0}^{2 n}\left(\nabla_{E_{i}} \phi\right) \phi E_{\imath}$, then

$$
\operatorname{div} V-\operatorname{div} U=r-r^{*}-S(\xi, \xi)+\frac{1}{2}|\nabla \phi|^{2}-\frac{3}{2}|d \Phi|^{2}-|\delta \Phi|^{2} .
$$

Proof. Compute

$$
\begin{aligned}
\operatorname{div} V=-\sum_{\imath, \jmath=0}^{2 n}\left\{g\left(\left(\nabla_{E_{\imath}} \phi\right) E_{\imath},\left(\nabla_{E_{j}} \phi\right) E_{\jmath}\right)\right. \\
\left.\quad+g\left(\left(R_{E_{j} E_{i}} \phi\right) E_{i}+\left(\nabla_{E_{i}} \nabla_{E_{j}} \phi\right) E_{\imath}, \phi E_{j}\right)\right\} \\
=-|\delta \Phi|^{2}+r-r^{*}-S(\xi, \xi)-\sum_{\imath, \jmath=0}^{2 n} g\left(\left(\nabla_{E_{i}} \nabla_{E_{j}} \phi\right) E_{\imath}, \phi E_{j}\right) .
\end{aligned}
$$

Moreover

$$
\begin{aligned}
\operatorname{div} U & =-\sum_{\imath, \jmath=0}^{2 n}\left\{g\left(\left(\nabla_{E_{j}} \nabla_{E_{i}} \phi\right) E_{\jmath}, \phi E_{\imath}\right)+g\left(\left(\nabla_{E_{\jmath}} \phi\right) E_{\imath},\left(\nabla_{E_{i}} \phi\right) E_{\jmath}\right)\right\} \\
& =-\sum_{\imath, \jmath=0}^{2 n} g\left(\left(\nabla_{E_{\jmath}} \nabla_{E_{i}} \phi\right) E_{\jmath}, \phi E_{\imath}\right)+\frac{3}{2}|d \Phi|^{2}-\frac{1}{2}|\nabla \phi|^{2},
\end{aligned}
$$


because of $|d \Phi|^{2}=\frac{1}{3}|\nabla \phi|^{2}-\frac{2}{3} \sum_{\imath, j=0}^{2 n} g\left(\left(\nabla_{E_{i}} \phi\right) E_{\jmath},\left(\nabla_{E_{j}} \phi\right) E_{\imath}\right)$. The equalities (5.3) and (5.4) give now our assertion, i. e. (5.2).

We know that any almost cosymplectic manifold satisfies necessarily the equalities (4.3) and (4.11). In a compact case the following theorem is true:

THEOREM 5.3. If a compact almost contact metruc manfold satrsfies the equalities (4.3) and (4.11), then it is almost cosymplectic.

Proof. By Green's theorem (cf. e.g. [3], Appendix 6), we have from (5.1) and (5.2)

$$
\begin{aligned}
& \int_{M}\left\{S(\xi, \xi)+|\nabla \xi|^{2}-(\delta \eta)^{2}-2|d \eta|^{2}\right\} d M=0, \\
& \int_{M}\left\{r-r^{*}-S(\xi, \xi)+\frac{1}{2}|\nabla \phi|^{2}-\frac{3}{2}|d \Phi|^{2}-|\delta \Phi|^{2}\right\} d M=0,
\end{aligned}
$$

respectively, where $d M$ is the natural volume element on $M$. These integrals under our assumptions, i. e. (4.3) and (4.11), reduce to

$$
\int_{M}\left\{(\delta \eta)^{2}+2|d \eta|^{2}\right\} d M=0, \quad \int_{M}\left\{\frac{3}{2}|d \Phi|^{2}+|\delta \Phi|^{2}\right\} d M=0 .
$$

Hence $d \eta=0$ and $d \Phi=0$, which completes the proof.

The forms $\eta$ and $\Phi$ of an almost cosymplectic manifold are coclosed, i.e. $\delta \eta=0$ and $\delta \Phi=0$ (see [2]; it also clearly follows from our relations (2.7) and (2.14)). Thus, they are harmonic forms. We prove

THEOREM 5.4. If an almost contact metric manifold $(M, \phi, \xi, \eta, g)$ fulfills the conditions (4.3), (4.11), $\nabla_{\xi} \xi=0$ and the forms $\eta$ and $\Phi$ are coclosed, then $(M, \phi, \xi, \eta, g)$ is almost cosymplectıc.

Proof. By our assumptions it follows from (5.1) that $d \eta=0$. The coclosedness of $\Phi$ yields immediately that $V=0$. On the other hand we have

$$
U=\sum_{i=0}^{2 n}\left(\nabla_{E_{i}} \phi^{2}\right) E_{i}-V=-\delta_{\eta} \xi+\nabla_{\xi} \xi-V=0 .
$$

The vanishing of vector fields $V, U$ and our assumptions used in (5.2) give $d \Phi=0$, completing the proof.

LEMMA 5.5. Any 3-dimensional almost contact metric manifold fulfils the relations $|\nabla \phi|^{2}=2|\nabla \xi|^{2}, 3|d \Phi|^{2}=2(\delta \eta)^{2}$ and $|\delta \Phi|^{2}=2|d \eta|^{2}$.

Proof. Choose a local $\phi$-basis $\left\{E_{0}=\xi, E_{1}, E_{2}=\phi E_{1}\right\}$ of $M$. By direct calculations we obtain 


$$
|\nabla \phi|^{2}=\sum_{\imath, \jmath, k=0}^{2}\left\{g\left(\left(\nabla_{E_{i}} \phi\right) E_{\jmath}, E_{k}\right)\right\}^{2}=2 \sum_{\jmath, k=0}^{2}\left\{g\left(\nabla_{E_{j}} E_{0}, E_{k}\right)\right\}^{2}=2|\nabla \xi|^{2} .
$$

Moreover one can verify that $3 d \Phi\left(E_{0}, E_{1}, E_{2}\right)=-\delta \eta, \delta \Phi\left(E_{0}\right)=2 d \eta\left(E_{1}, E_{2}\right), \delta \Phi\left(E_{1}\right)$ $=2 d \eta\left(E_{2}, E_{0}\right), \delta \Phi\left(E_{2}\right)=2 d \eta\left(E_{0}, E_{1}\right)$. These equations give $|d \Phi|^{2}=2 / 3(\delta \eta)^{2}$ and $|\delta \Phi|^{2}=2|d \eta|^{2}$, completing the proof.

The following corollaries one can obtain as consequences of the above lemma.

COROllary 5.6. Any 3-dimensional almost contact metric manifold is cosymplectic if and only if $\nabla \xi=0$.

COROLlary 5.7. Let $(M, \phi, \xi, \eta, g)$ be a 3-dimensional almost contact metruc manifold. If at least one of the forms $\eta$ or $\Phi$ is harmonic, then $(M, \phi, \xi, \eta, g)$ is almost cosymplectic.

The next theorem follows from Lemma 5.1 and Corollary 5.7.

THEOREM 5.8. Any compact 3-dimensional almost contact metruc manifold satisfying the condition (4.11) is almost cosymplectic.

\section{$\S 6$. Almost cosymplectic manifolds under some additional conditions.}

It has been proved in [1] that a cosymplectic manifold of constant curvature is locally flat. For almost cosymplectic manifolds it is proved in [2] that an almost cosymplectic manifold of constant curvature is cosymplectic if and only if it is locally flat. However we prove here

THEOREM 6.1. Almost cosymplectic manifolds of non-zero constant curvature do not exist in dimensions $\geqq 5$.

Proof. Assume $R_{X Y} Z=K\{g(Y, Z) X-g(X, Z) Y\}, K=$ const. $\neq 0$. Then (4.9) and (4.10) give

$$
\begin{aligned}
\left(\nabla_{\nabla Z \xi} \phi\right) X & =K\{\eta(X) \phi Z-g(\phi Z, X) \xi\}, \\
g\left(\nabla_{Y} \xi, \nabla_{Z} \xi\right) & =-K\{g(Y, Z)-\eta(Y) \eta(Z)\},
\end{aligned}
$$

respectively. Since by (2.5) $g\left(\nabla_{Y} \xi, \nabla_{Z} \xi\right)=g\left(\nabla_{\nabla_{Z} \xi} \xi, Y\right)$, we obtain from (6.2) $\nabla_{\nabla Z \xi} \xi$ $=-K\{Z-\eta(Z) \xi\}$. Therefore, the substitution $\nabla_{Z} \xi$ instead of $Z$ in (6.1) yields, by (2.10),

$$
\left(\nabla_{Z} \phi\right) Y=-\eta(X) \phi \nabla_{Z} \xi-g\left(\nabla_{Z} \xi, \phi X\right) \xi .
$$

Hence we derive $|\nabla \phi|^{2}=2|\nabla \xi|^{2}$. But $|\nabla \xi|^{2}=-2 n K$, by (4.11). Thus, $|\nabla \phi|^{2}=$ $-4 n K$. In view of this relation and $r=2 n(2 n+1) K, r^{*}=2 n K, S(\xi, \xi)=2 n K$, it follows from (4.3) that $n=1$ or $\operatorname{dim} M=3$. This completes the proof. 
THEOREM 6.2. Let $(M, \phi, \xi, \eta, g)$ be a conformally flat almost cosymplectic manifold with $\operatorname{dim} M \geqq 5$. Then the scalar curvature of $(M, \phi, \xi, \eta, g)$ is nonpositive and the manifold is cosymplectic if and only of it is locally flat.

Proof. Conformal flatness yields

$$
\begin{gathered}
R_{X Y Z W}=\frac{1}{2 n-1}\{g(X, W) S(Y, Z)-g(Y, W) S(X, Z)+g(Y, Z) S(X, W) \\
-g(X, Z) S(Y, W)\}-\frac{r}{2 n(2 n-1)}\{g(X, W) g(Y, Z)-g(X, Z) g(Y, W)\} .
\end{gathered}
$$

It follows immediately from (6.3) that $r^{*}=\frac{1}{2 n-1}\{r-2 S(\xi, \xi)\}$, which together with (4.3) and (4.11) gives

$$
\frac{2 n-2}{2 n-1} r+\frac{2 n-3}{2 n-1}|\nabla \xi|^{2}+\frac{1}{2}|\nabla \phi|^{2}=0 \text {. }
$$

From (6.4) we have $r \leqq 0$ and $r=0$ if and only if $\nabla \xi=0, \nabla \phi=0$. To finish the proof we must only prove that cosymplecticity of $(M, \phi, \xi, \eta, g)$ implies its local flatness. But, if $\nabla \xi=0$ and $\nabla \phi=0$, then $R_{X Y} \xi=0$ and by (6.4) $r=0$. Hence $S(X, \xi)=0$. Substituting $X=W=\xi$ in (6.3) and applying the above relations we obtain $S(Y, Z)=0$, which completes the proof.

In the next theorem we consider an almost cosymplectic manifold of constant $\phi$-sectional curvature. That is, we assume that at any point $p \in M$ the sectional curvature $K(X, \phi X)$ (denote it by $H_{p}$ ) is independent of the choice of the tangent vector $X \in T_{p}(M), 0 \neq X \perp \xi$. By $H$ we denote the $\phi$-sectional curvature of the manifold, i. e. $H: M \rightarrow \boldsymbol{R}, H(p)=H_{p}$.

THEOREM 6.3. Let $(M, \phi, \xi, \eta, g)$ be an almost cosymplectic manifold of constant $\phi$-sectional curvature. Then the scalar curvature and the $\phi$-sectional curvature satisfy the inequality $n(n+1) H \geqq r$. Equality holds if and only if $(M, \phi, \xi, \eta, g)$ is cosymplectic.

Proof. By the assumption we have $R_{X_{\phi} X X_{\phi} X}+H_{p}|X|^{4}=0$ at any point $p \in M$ and for any $X \in T_{p}(M), X \perp \xi$. Clearly, this condition implies

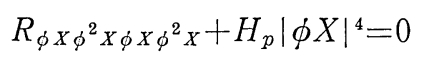

at any point $p \in M$ and for any $X \in T_{p}(M)$. Set

$$
P_{X Y Z W}=R_{\phi X \phi^{2} Y \dot{\phi} Z_{\dot{\rho}}{ }^{2} W}+H_{p} g(\phi X, \phi Z) g(\phi Y, \phi W) \text {. }
$$

The tensor $P$ satisfies $P_{X Y Z W}=P_{Z W X Y}$. Therefore (6.5) is equivalent to

$$
\begin{aligned}
& P_{X Y Z W}+P_{X Y W Z}+P_{Y X Z W}+P_{Y X W Z}+P_{X W Y Z}+P_{X W Z Y} \\
& \quad+P_{W X Y Z}+P_{W X Z Y}+P_{X Z Y W}+P_{X Z W Y}+P_{Z X Y W}+P_{Z X W Y}=0 .
\end{aligned}
$$


Choosing a $\phi$-basis, taking $X=W=E_{\imath}, Y=Z=E$, into (6.6) and summing over $i$ and $j$ we obtain

$$
\sum_{\imath, \jmath=0}^{2 n}\left(P_{E_{i} E_{j} E_{j} E_{\imath}}+P_{E_{i} E_{j} E_{i} E_{j}}+P_{E_{i} E_{i} E_{j} E_{j}}\right)=0,
$$

which by the definition of $P$ and the first Bianchi identity gives

$$
4 n(n+1) H-3 r *-r+2 S(\xi, \xi)=0 .
$$

This together with (4.3) and (4.11) yields

$$
n(n+1) H-r=\frac{5}{4}|\nabla \xi|^{2}+\frac{3}{8}|\nabla \phi|^{2},
$$

which completes the proof.

\title{
REFERENCES
}

[1] D.E. Blair, The theory of quası-Sasakıan structures, J. Differential Geometry, 1 (1967), 331-345.

[2] S. I. GoldBerg AND K. YANO, Integrability of almost cosymplectic structures, Pacific Journal of Mathematics, 31 (1969), 373-382.

[3] S. Kobayashi and K. Nomizu, Foundations of differential geometry, Vol. I, Interscience Publishers (1963).

[4] K. YANO, Integral formulas in Riemannian geometry, Marcel Dekker, INC. (1970).

\author{
Institute of Mathematics \\ TECHNICAL UNIVERSITY \\ Wroceaw, POLAND
}

